\title{
Looking at CER from Medicare's Perspective
}

\author{
Penny Mohr, MA
}

\begin{abstract}
BACKGROUND: Comparative effectiveness research (CER) is rapidly adding to the amount of data available to health care coverage and payment decision makers. Medicare's decisions have a large effect on coverage and reimbursement policies throughout the health insurance industry and will likely influence the entire U.S. health care system; thus, examining its role in integrating CER into policy is crucial.
\end{abstract}

OBJECTIVES: To describe the potential benefits of CER to support payment and coverage decisions in the Medicare program, limitations on its use, the role of the Centers for Medicare \& Medicaid Services (CMS) in improving the infrastructure for CER, and to discuss challenges that must be addressed to integrate CER into CMS's decision-making process.

SUMMARY: A defining feature of CER is that it provides the type of evidence that will help decision makers, such as patients, clinicians, and payers, make more informed treatment and policy decisions. Because CMS is responsible for more than $\mathbf{4 7}$ million elderly and disabled beneficiaries, the way that Medicare uses CER has the potential to have a large impact on public and individual health. Currently many critical payment and coverage decisions within the Medicare program are made on the basis of poorquality evidence, and CER has the potential to greatly improve the quality of decision making. Despite common misconceptions, CMS is not prohibited by law from using CER apart from some reasonable limitations. CMS is, however, required to support the development of the CER infrastructure by making their data more readily available to researchers. While CER has substantial potential to improve the quality of the agency's policy decisions, challenges remain to integrate CER into Medicare's processes. These challenges include statutory ambiguities, lack of sufficient staff and internal resources to take advantage of CER, and the lack of an active voice in setting priorities for CER and study design.

CONCLUSION: Although challenges exist, CER has the potential to greatly enhance CMS's ability to make decisions regarding coverage and payment that will benefit both the agency and their patient population.

J Manag Care Pharm. 2012;18(4-a):S5-S8

Copyright $\odot 2012$, Academy of Managed Care Pharmacy. All rights reserved.

\section{What is already known about this subject}

- Without comparative effectiveness research (CER), payers and Centers for Medicare \& Medicaid Services (CMS) must make decisions based on the best evidence available, which often lacks headto-head data comparison, uses surrogate endpoints, and excludes the specific elderly or disabled population that Medicare covers.

- There is a common misperception that the CMS is not allowed to use CER data when making decisions.

\section{What this article adds}

- Contrary to a common misperception, CMS is allowed to use CER data when making coverage decisions, albeit with some limitations.

- CER could be used when creating patient decision aids, establishing value-based insurance design, and in coding (determining whether there is enough of a significant therapeutic distinction for a particular product to assign it a new code).

- CMS could use CER data in pricing decisions-specifically in the area of least-costly alternative (or reference pricing).

\section{What this article adds (continued)}

- Greater use of Coverage with Evidence Development is a mechanism to stimulate the generation of relevant CER for the Medicare program.

- Some possible impediments to integrating more CER into CMS decisions may be a lack of training in how to utilize observational data, as well as an inadequate number of epidemiologists on staff who can manage the future increase in volume of CER data.

- This article makes arguments for why CMS should have a voice in setting priorities for research funded by the Patient-Centered Outcomes Research Institute (PCORI), so that the resulting research will be meaningful to CMS decision makers.

$\mathrm{W}$ ith responsibility for more than 47 million elderly and disabled beneficiaries, ${ }^{1}$ the way that Medicare uses comparative effectiveness research (CER) has the potential to have a large impact on public and individual health. Medicare's decisions also have a much larger effect on coverage and reimbursement policies throughout the health insurance industry, ${ }^{2}$ and these effects will likely extend throughout the U.S. health care system. For this reason, it is critical to understand how Medicare might use CER. This article examines CER from the Medicare program's perspective, including the program's current use of CER, common misconceptions about the ability of the Centers for Medicare \& Medicaid Services (CMS) to use CER, and the role CMS has in future CER development.

\section{The Lack of CER Impedes Effective Decision Making}

Medicare and, ultimately, every payer in the health care system have the same fundamental questions when it comes to making decisions about paying for the drugs, devices or other services they cover: "How does this technology work in my population under circumstances of normal practice, and does it provide meaningful health benefits compared with existing treatments over the long term?" The italicized terms represent the gaps in existing clinical evidence that CER is designed to fill. Without data that address these questions, payers (including CMS), must make decisions based on studies that often have used poorly validated surrogate endpoints, have been conducted in carefully selected populations and settings, or may include no relevant treatment comparators.

The quality of information CMS commonly uses for decision making is illustrated by a systematic review of the efficacy of offlabel use for 19 approved cancer drugs conducted by the Duke Evidence-based Practice Center, published in 2010. After reviewing several thousand trials, the authors concluded: "Because of the paucity of high-quality evidence, the data available — though voluminous-may have little meaning or value for informing clinical practice." 3

Without doubt, the paucity of high-quality evidence has direct ramifications on the performance of the Medicare program. The 
area of decision making around prostate cancer treatments provides a good example. Prostate cancer is the leading cause of cancer and the second leading cause of mortality among men. ${ }^{4}$ While there are many alternatives for treating prostate cancer, there is a lack of robust evidence about which treatments work best at what stage of the disease. ${ }^{5}$

In 2002, CMS covered intensity modulated radiation therapy (IMRT) on the basis of limited information about its performance relative to brachytherapy (which was the standard in use at the time). At the time, the cost of IMRT was about double that of brachytherapy. ${ }^{6}$ Within a few years, roughly one-third of beneficiaries were using IMRT, and expenditures were well over a billion dollars for that particular technology. ${ }^{7}$

More recently, proton beam therapy was introduced, and Medicare contractors made the decision to cover it. This essentially doubled Medicare expenditures again for a course of radiation therapy for prostate cancer, but still without good evidence that it improves the quality of care over IMRT or even over brachytherapy. We still do not know which therapies are more effective for which patients and which stages of prostate cancer, but treatment costs for this condition continue to escalate. Unfortunately for prostate cancer patients, these types of decisions not only affect their out-of-pocket obligations, but also could result in exposing them to undue toxicities and risk.

A defining feature of CER that makes it fundamentally different from the way clinical research has been done in the past is its purpose. According to the Institute of Medicine (IOM), "The purpose of CER is to assist consumers, clinicians, purchasers, and policy makers to make informed decisions that will improve health care at both the individual and population levels." 8 To this effect, CER provides unique opportunities for CMS to improve its policies to ensure medical services are used more appropriately.

\section{Can CMS Legally Use CER?}

There is a common misperception that CMS is not allowed to use CER results when making decisions. The truth is that CMS is allowed to use CER data, albeit with some limitations. The limitations imposed on CMS's use of CER are fairly modest. CMS must use an iterative and transparent process, and they cannot use CER as a sole source of information. ${ }^{9}$ The Patient Protection and Affordable Care Act (ACA) ${ }^{9}$ of 2010 includes specific limitations on Medicare's use of CER..$^{10}$ CMS can't use it in a manner that treats the life extension of the elderly, disabled, or terminally ill differently than others, and they can't use it to preclude or discourage choices between extending life or risk of disability. ${ }^{10}$ Additionally, CMS can't include mandates for coverage or payment, cannot use cost per quality adjusted life years threshold to determine coverage or payment and must use evidence only as part of a larger process. ${ }^{10}$ In fact, these limitations reflect the way CMS already makes coverage decisions, and these limitations do not fundamentally change CMS's current processes.

The larger question is how these limitations from the ACA affect CMS's ability to use costs. The Patient-Centered Outcomes Research Institute (PCORI), a public/private entity charged with identifying research priorities, establishing an agenda, and fund- ing future CER studies, created under the ACA, ${ }^{9}$ cannot fund research or advocate for a threshold for quality-adjusted life years (QALYs). In addition, the ACA imposed a restriction that the Secretary of Health and Human Services, acting for the Medicare program, could not use a "threshold" for QALYs in their coverage determinations. CMS has gone on record in guidance documents stating that they don't use cost or cost-effectiveness in their decision making. This restriction against using a threshold of QALYs is not a new limitation for CMS, but it is now incorporated into statute.

Another important aspect of the ACA is that it explicitly allows CMS to use differential copayments based on cost, which could open the potential of using value-based insurance design within the CMS program.

\section{CMS's Obligation to CER: Making Data Available for Research}

In recent years, significant federal funds have been expended in building an infrastructure to support CER, such as registries and claims databases. One of the legislative requirements is that CMS must make its data available for PCORI-sponsored research. Although CMS data have been available for a long time, the data have been relatively difficult to obtain. This new requirement may facilitate researcher access.

To that end, one of the major investments has been the development of the Multi-Payer Claims Database (MPCD). ${ }^{11}$ The database combines Medicare data, including the institutional claims in Part A, outpatient claims from Part B, and the drug data from Part D, with claims data from private insurers. This potentially will deliver a much more robust observational data set for doing CER. Currently, 1 private payer is contributing claims data and hopefully more insurers will contribute data over time. Medicare data provide a rich resource for CER

Using existing Medicare data, as with other claims data, is challenging. It is not possible to determine factors that contribute to treatment decisions, patients are not randomly assigned to treatment, and selection bias is an issue. These could be resolved if beneficiaries were randomly assigned to plans with different formularies based on factors such as low income subsidies. ${ }^{12}$

However, much work remains in determining how to implement this requirement. Some of the outstanding questions include: How should a public/private partnership like this be sustained? Should there be benefits to the participants who add their private claims to the database, and if so, what type of benefits? Who will own the data? And how should the data be made available to researchers? Another important question is whether these data will be linkable to clinically rich information from randomized controlled trials or electronic health records, which could potentially make this a much more powerful and robust source.

Another outstanding question is whether this investment will result in observational data becoming more acceptable for coverage decision making. Currently, observational data are seldom used for making Medicare national coverage determinations. Some possible impediments to integrating observational data into coverage decisions may be a strong existing bias that randomized controlled trials (RCTs) are the only acceptable method 
Looking at CER from Medicare's Perspective

TABLE 1 Comparison of CMS's Top 20 Research Priorities with IOM, CER and NIH Challenge Grant Priorities

\begin{tabular}{|c|c|c|c|}
\hline Disease Area & CMS Evidentiary Priorities & IOM & $\mathrm{NIH}$ \\
\hline Oncology/Hematology & Appropriate ESA Use in Cancer Patients & & \\
\hline Oncology/Hematology & Benefits of Cancer Prognostic Markers & $\boldsymbol{V}$ & \\
\hline Oncology/Hematology & Benefits of High-Cost Cancer Drugs & & \\
\hline Oncology/Hematology & New Radiation Treatments for Cancer (e.g., proton beam, IMRT) & $\checkmark$ & \\
\hline Cardiovascular Disease & Treatment of Atrial Fibrillation & $\checkmark$ & $\checkmark$ \\
\hline Cardiovascular Disease & Does Screening for Atherosclerotic Disease Improve Health Outcomes? & & \\
\hline Cardiovascular Disease & Effectiveness of CT Angiography & $\checkmark$ & \\
\hline Cardiovascular Disease & Prevention of Congestive Heart Failure & $\checkmark$ & $\checkmark$ \\
\hline Diabetes & Benefit of Early Aggressive Treatment for Diabetes & $\checkmark$ & $\checkmark$ \\
\hline Diabetes & Comparative Effectiveness of All Diabetes Treatments Using Hard Outcomes & $\checkmark$ & \\
\hline Diabetes & Benefit of Weight Loss Medication on Diabetes & $\checkmark$ & $\checkmark$ \\
\hline Diabetes & Optimal Hemoglobin Alc Goals in the Elderly & $\checkmark$ & $\checkmark$ \\
\hline Other & Appropriate Use of Hospice Care & & \\
\hline Other & Appropriate End-of-Life Care & $\checkmark$ & $\checkmark$ \\
\hline CNS/Neurology/Behavior & Improving Depression Care in Primary Care & $\checkmark$ & \\
\hline CNS/Neurology/Behavior & Appropriate Treatment of Carotid Artery Disease & & $\checkmark$ \\
\hline CNS/Neurology/Behavior & Comparative Effectiveness of Acute Stroke Treatment: Clot Retrieval vs. Reperfusion Drugs & & \\
\hline CNS/Neurology/Behavior & Comparative Effectiveness of Treatment of Intracranial Disease & & \\
\hline Wound Care & Comparative Effectiveness of Treatment for Ulcers: Off-loading, Debridement, Biologics, Revascularization & $\checkmark$ & $\boldsymbol{V}$ \\
\hline
\end{tabular}

for answering questions about relative effectiveness and an inadequate number of epidemiologists on staff who understand how to utilize observational data.

\section{A Disconnect: The Lack of a Voice in Setting Priorities for CER Investment}

CER will be credible, timely, and relevant to Medicare beneficiaries only if CMS becomes a more active participant in defining research priorities. The agency needs comparative studies that address issues specific to the elderly, chronically ill, and disabled populations that it serves. When comparing the top 20 priorities established by CMS in 2007 with those established by the IOM and those established by the National Institutes of Health (NIH) for NIH Challenge Grants, ${ }^{13}$ there are some notable differences (Table 1). Specifically, CMS was interested in looking at the impact of expensive cancer drugs, the use of reperfusion drugs for stroke prevention, and erythropoietin-stimulating agents and their use in cancer patients.

The Medicare program, either by design or by default, does not currently have an active voice in establishing priorities for CER investment. Although the legislation allocated space on PCORI's governing board for 2 federal or state representatives, ${ }^{9} \mathrm{CMS}$ is not represented. If CMS doesn't have a seat at the table in PCORI and if there's no effective mechanism for CMS to influence the types of studies being done, potentially there may be major gaps in the type of relevant and critical information that they need.

\section{The Future of CER in the Medicare Program}

CER has the potential for broad usage throughout the Medicare program. Not only could the results be used to inform coverage decisions, but they could also be used to support patient decision aids and assist in coding decisions (determining whether there is enough of a significant therapeutic distinction for a particular product to assign it a new code). In addition, and perhaps even more importantly for CMS, CER data could be used in pricing-specifically in the area of developing policies for value-based insurance design and least-costly alternative pricing (or reference pricing). Through least-costly alternative pricing, Medicare contractors have the discretion to use relative costs in setting payments if there are 2 alternative items with equal efficacy. This has been used primarily to set payments for durable medical equipment. While a recent legal ruling has restricted use of this policy for drugs, this approach could help promote the generation of better CER for Medicare decisions. ${ }^{14}$ For example, in the prostate cancer case, if research couldn't demonstrate a significant clinical difference between proton beam therapy and intensity modulated radiation therapy, then CMS could pay the same for those treatments rather than paying different amounts for each. Pearson and Bach suggest a framework where new technology would be paid for at a higher rate than existing technology for 3 years. At the end of the third year, cost for the new technology would be reduced to the existing technology cost unless the new technology demonstrated clinical advantages to justify the additional cost. ${ }^{10}$

Another policy tool the agency has to help promote the development of CER is Coverage with Evidence Development (CED), also referred to as conditional coverage. CED links reimbursement to the requirement for prospective data collection. This policy tool allows CMS to help drive clinical research to ensure it is more relevant to its needs, such as ensuring that the 
population researched in the studies includes Medicare beneficiaries, and that the outcomes include significant clinical outcomes that are relevant to the beneficiaries. CMS has been involved in more than a dozen CED decisions over the last 15 years. ${ }^{13}$ Although there was a brief hiatus in these types of decisions, activity has recently increased.

For many of the policies that are mentioned above, there are statutory ambiguities that keep the agency from being as proactive as they could be to use and promote the development of CER.

\section{Conclusions}

Currently, the quality of existing evidence has often been a limitation for CMS in its decision making. CER has the potential to improve the quality of that evidence. However, there are some key challenges to address before CMS can use CER throughout the program. One challenge is a lack of sufficient staff and internal resources devoted to take advantage of CER. Another challenge to tying CER to reimbursement programs, such as least-costly alternative, is the long-standing institutional separation between the areas of coverage and payment policy, which would have to be overcome. In addition, statutory ambiguities inhibiting CMS's ability to use policies such as CED or least-costly alternative are limiting CMS's potential to be a value-based purchaser, and at least some of those statutory barriers must be corrected before the agency can use CER effectively.

In the end, CMS needs to be more effectively engaged in establishing research priorities to ensure that at least some of the CER data are relevant to their needs. CMS must find a way to make those needs transparent and become involved in determining research priorities.

\section{Author}

PENNY MOHR, MA, is Vice President, Program Development, Center for Medical Technology Policy, Baltimore, Maryland.

AUTHOR CORRESPONDENCE: Penny Mohr, MA; Center for Medical Technology Policy, 401 E. Pratt St., Ste. 631, Baltimore, MD 21202. Tel.: 410.547 .2687 x114;

E-mail:Penny.Mohr@cmtpnet.org.

\section{DISCLOSURES}

This supplement was sponsored by PIM and StrataMed through an educational grant from Novo Nordisk. Penny Mohr received compensation from PIM for participating in the live continuing education activity on which this article is based and for writing the article. Mohr reports payment for contracted research with the National Pharmaceutical Council.

\section{ACKNOWLEDGEMENTS}

The author thanks Kelley J. P. Lindberg, BS, for writing assistance in preparation of this manuscript.

\section{REFERENCES}

1. The Henry J. Kaiser Family Foundation. Total number of Medicare beneficiaries, 2011. Available at: http://www.statehealthfacts.org/comparemaptable. jsp?ind=290\&cat=6. Accessed February 29, 2012.

2. Gornick ME, Warren JL, Eggers PW, et al. Thirty years of Medicare: impact on the covered population. Health Care Financ Rev. 1996;18(2):179237. Available at: http://ssa.gov/history/pdf/ThirtyYearsPopulation.pdf. Accessed February 29, 2012.

3. Abernethy AP, Coeytaux RR, Carson, K, et al.; Duke Evidence-based Practice Center. Report on the evidence regarding off-label indications for targeted therapies used in cancer treatment. Technology assessment report. Project ID CANT1106. Agency for Healthcare Research and Quality. January 29, 2010. Available at: http://www.cms.gov/determinationprocess/downloads/id71TA.pdf. Accessed March 15, 2012.

4. U.S. Department of Health and Human Services, Centers for Disease Control and Prevention. United States cancer statistics: 1999-2007 cancer incidence and mortality data. National Cancer Institute. 2010. Available at: http://www.cdc.gov/uscs. Accessed January 24, 2012

5. Wilt TJ, Shamliyan T, Taylor B, et al; Minnesota Evidence-based Practice Center. Comparative effectiveness of therapies for clinically localized prostate cancer. Comparative effectiveness review no. 13. Agency for Healthcare Research and Quality. Publication no. 08-EHC010-1. February 2008. Available at: http://www.effectivehealthcare.ahrq.gov/index.cfm/search-forguides-reviews-and-reports/?pageaction=displayproduct\&productid=79. Accessed January 24, 2012

6. Ollendorf DA, Hayes J, McMahon P, Kuba M, Pearson SD. Management options for low-risk prostate cancer: a report on comparative effectiveness and value. Boston, MA: Institute for Clinical and Economic Review. December 2009. Available at: http://www.icer-review.org/index.php/Reports/ lrpc.html. Accessed January 24, 2012.

7. Carreyrou J, Tamman M. A device to kill cancer, lift revenue. Wall Street J. December 7, 2010. Available at: http://online.wsj.com/article/SB10001424052 748703904804575631222900534954.html. Accessed January 24, 2012.

8. Committee on Comparative Effectiveness Research Prioritization, Board on Health Care Services, Institute of Medicine of the National Academies. Initial National Priorities for Comparative Effectiveness Research. Washington, DC: The National Academies Press; 2009. Available at: http://www.iom.edu/ Reports/2009/ComparativeEffectivenessResearchPriorities.aspx. Accessed February 15, 2011.

9. Patient Protection and Affordable Care Act. H.R. 3590, 11lth Cong., 2nd Sess. (2010). Available at: http://www.gpo.gov/fdsys/pkg/PLAW-11lpubll48/ pdf/PLAW-111publ148.pdf. Accessed January 24, 2012.

10. Pearson SD, Bach PB. How Medicare could use comparative effectiveness research in deciding on new coverage and reimbursement. Health Aff. 2010;29(10):1796-804.

11. Conway PH, VanLare JM. Improving access to healthcare data: the open government strategy. JAMA. 2010;304(9):1007-08.

12. Fung V, Brand RJ, Newhouse JP, Hsu J. Using Medicare data for comparative effectiveness research: opportunities and challenges. Am J Manag Care. 2011;17(7):489-96. Available at: http://www.ajmc.com/publications/ issue/2011/2011-7-vol17-n7/AJMC_1ljul_Fung_488to496/2. Accessed March 27, 2012.

13. Mohr PE, Tunis S, Sabharwal R, Montgomery R, Bergthold L. The comparative effectiveness research landscape in the United States and its relevance to the Medicare program. Prepared for the Medicare Payment Advisory Commission. May 2010. Available at: http://htaiced.files.wordpress. com/2011/02/cer-and-ced-in-medicare.pdf. Accessed January 30, 2012.

14. Hays v. Leavitt. U.S. District Court for the District of Columbia. 1:2008cv01032 (October 16, 2008). Available at: http://dc.findacase.com/ research/wfrmDocViewer.aspx/xq/fac.\%2FFDCT\%2FDDC\%2F2008\% 2F20081016_0001011.DDC.htm/qx. Accessed January 30, 2012. 